\title{
Prevalence of intestinal parasitic infections among patients attending Bugando Medical Centre in Mwanza, north-western Tanzania: a retrospective study
}

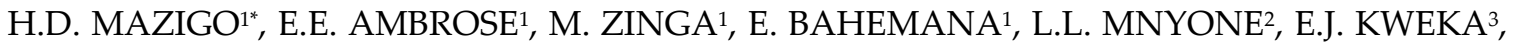 \\ and J. HEUKELBACH ${ }^{4}$ \\ ${ }^{1}$ Department of Medical Parasitology and Entomology, Weill-Bugando University College of Health \\ Sciences, P.O. Box 1464, Mwanza, Tanzania \\ ${ }^{2}$ Sokoine University of Agriculture, Pest Management Centre, Morogoro, Tanzania \\ ${ }_{3}^{3}$ Tropical Pesticide Research Institute, Arusha, Tanzania, \\ ${ }^{4}$ Department of Community Health, School of Medicine, Federal University of Ceará, Fortaleza, Brazil
}

\begin{abstract}
Gastrointestinal helminths and protozoan parasites may cause mild, acute and chronic human infections. There is inadequate reliable information on the epidemiology of these parasites among patients attending tertiary hospitals in Tanzania. This retrospective study was conducted using hospital data obtained from the Department of Medical Parasitology of Bugando Medical Centre (BMC) in Mwanza, Tanzania. A total of 3152 stool samples were recorded from January 2008-March 2010. Intestinal parasitic infections were recovered in $57.1 \%$ (1799/3152) of the stool samples. Helminths eggs were observed in $36.6 \%(1,153 / 3,152)$ of the samples with hookworm eggs recovered in $25.2 \%(793 / 3125)$ and S. mansoni in 5.6\% (177/3125) of the samples. Protozoan parasites were recovered in 20.5\% (646/3152) of the samples in which $13.6 \%$ 428/3152) had Entamoeba histolytical E. dispar and 6.9\% (218/3152) Giardia lamblia. Prevalence of intestinal helminth infections was higher in females, 55.7\% (95CI\%, 51.7-59.7, $\mathrm{n}=642)$ than males $(\mathrm{n}=511$, $44.3 \%, 95 \% \mathrm{CI}, 40.3-48.6)$. Similarly, the prevalence of protozoan infections was significantly higher among females (58.1\%, 95\%CI, 53.1-63.1) than males (42.3\%, 95\%CI, 36.5-48.2). The prevalence of helminth infections was highest among $45+$ years olds and lowest in the $0-4$ years olds while that of protozoan infections was highest in the 5-14 years age group $(30.9 \%, 95 \% \mathrm{CI}$, 27.9-33.8) and lowest in the 0-4 years age group. In conclusion, this study shows that intestinal helminth and protozoan infections are among the common parasitic infections among patients presenting at BMC. This information may provide invaluable statistics needed for planning meaningful public control programmes that aim at reducing the prevalence and morbidity of parasitic infections.
\end{abstract}

Keywords: helminth, protozoa, parasitic infections, prevalence, hospital, Tanzania

\section{Introduction}

Intestinal helminths and protozoan parasites are major public health problems in developing countries (Lwambo et al., 1999; Handzel at al., 2003; Bethony et al., 2002). These infections have common characteristics - they are highly endemic in populations with low socio-economic status and poor hygiene, favouring larval skin penetration and oral-faecal transmission (Ravdin, 1995; Hotez et al., 2004; Sayyari et al., 2005, Bethony et

\footnotetext{
Correspondence: *Dr. Humphrey D. Mazigo: humphreymazigo@gmail.com
} 
al., 2006). The parasites are important causal agents of gastrointestinal disorders such as diarrhoea, dysentery, vomiting, lack of appetite, haematuria, abdominal distension and sometimes mentally related disorders (Garcia, 2004; Bethony et al., 2006). Moreover, heavy chronic infections with Ascaris lumbricoides and hookworms (Ancylostoma doudenale or Necator americanus) may cause malnutrition and anaemia in high risk groups (Albanico et al., 1998; Awasti et al., 2003; Hotez et al., 2004). Chronic infections with Schistosoma species (S. haematobium and S. mansoni in Tanzania) have been associated with hepatomegaly, splenomegaly, periportal fibrosis, hypertension, urinary bladder obstructions, cancer and tumour of the prostate glands (Boros, 1989; Guyatt et al., 1999; Haidar, 2001; Malenganisho et al., 2008; Mazigo et al., 2010a).

In northwest Tanzania, there is inadequate information on the magnitude of parasitic infection among cases attended at tertiary hospitals. It has been described that information generated from in-patients departments of hospitals may provide the initial information needed for planning meaningful public control programmes (Obiamiwe, 1972). In addition, differentiation of parasitic agents is an important step for initiation of tailored treatment and prevention strategies. Therefore, the present retrospective study was conducted to determine the species and prevalence of human intestinal parasites among in-patients at Bugando Medical Centre in Mwanza, Tanzania.

\section{Materials and Methods}

\section{Study area}

The study was conducted at Bugando Medical Centre in Mwanza, north-western Tanzania. This referral hospital is situated along the southern shores of Lake Victoria and has a 900 bed-capacity. BMC is located between latitudes $2^{\circ} 15^{\prime}-2^{\circ} 45^{\prime} S$ and longitudes $32^{\circ} 45^{\prime}-45^{\circ} 38^{\prime} \mathrm{E}$ and lies at an altitude of $1140 \mathrm{~m}$. The hospital serves as a referral centre for tertiary specialist care for a catchment population of approximately 13 million people from Mwanza, Mara, Kagera, Shinyanga, Tabora and Kigoma regions of Tanzania (http://www.bugandomedicalcentre.go.tz).

\section{Data collection and analysis}

The study examined sample records of laboratory results contained in a designed computer database. The data covered the period of 27 months from January 2008 to March 2010. The routine stool examination method was a direct smear and $10 \%$ formolether concentration technique (WHO, 1991). All stool sample records were examined and positive results for helminths eggs, larvae and protozoan cyst or trophozoite infections were recorded.

Variations in distribution patterns of positive stool samples between sex and age were determined. The prevalence of infections was reported in proportions. Chi-square test $\left(\chi^{2}\right)$ was used to compare relative frequencies between groups (sex and age). Data analysis was conducted using SPSS version 11.5 (SPSS Inc, Chicago, Illinois).

\section{Ethical considerations}


Ethical clearance to conduct this study was obtained from the Bugando Medical Centre Ethical Committee (Ref: AB.286/317/01/41).

\section{Results}

A total of 3152 stool samples (1887 in 2008, 963 in 2009 and 302 in 2010) were submitted to the Department of Medical Parasitology of BMC and included in the analysis. Of these, $1153(36.6 \%$; 95\%CI, 33.9-39.4) were positive for intestinal helminths and 646 (20.5\%, 95\%CI, 17.4-23.6) for intestinal protozoa. Hookworm accounted for the most prevalent parasitic infection $(25.2 \%(95 \% \mathrm{CI}, 22.2-28.2 ; \mathrm{n}=793)$ followed by Schistosoma mansoni, 5.6\% (95\%CI, 2.2-9; $\mathrm{n}=177$ ) (Table 1). Entamoeba histolytical E. dispar $(13.6 \%$, 95\%CI, 6.6-20.8) and Giardia lamblia (6.9\%, 95\%CI,-0.001-14.3) were the only intestinal protozoan observed in the study (Table 1).

Table 1: Prevalence (\%) of helminth and protozoan infections, March 2008-Jan 2010

\begin{tabular}{llllll}
\hline Parasite & $\mathbf{2 0 0 8}$ & $\mathbf{2 0 0 9}$ & $\mathbf{2 0 1 0}$ & $\begin{array}{l}\text { Overall } \\
\text { prevalence }\end{array}$ & P-value \\
\hline Helminth infections & & & & & \\
Hookworms & $274(14.5 \%)$ & $414(42.9 \%)$ & $105(34.8 \%)$ & $793(25.2 \%)$ & $<0.004$ \\
A. lumbricoides & $50(2.6 \%)$ & 0.00 & 0.00 & $50(1.6 \%)$ & $<0.05$ \\
E. vermicularis & $9(0.5 \%)$ & $10(1.0 \%)$ & $2(0.7 \%)$ & $21(0.67 \%)$ & $<0.06$ \\
T. trichiura & $8(0.4 \%)$ & $13(1.4 \%)$ & $4(1.3 \%)$ & $25(0.79 \%)$ & $<0.07$ \\
S. mansoni & $46(2.4 \%)$ & $103(10.7 \%)$ & $28(9.3 \%)$ & $177(5.6 \%)$ & $<0.003$ \\
Taenia species & $4(0.2 \%)$ & $13(1.3 \%)$ & $1(0.3 \%)$ & $18(0.57 \%)$ & $<0.06$ \\
Protozoan infections & & & & & \\
E. histolytica/E. dispar & $31(1.6 \%)$ & $271(28.1 \%)$ & $126(41.7 \%)$ & $428(13.6 \%)$ & $<0.001$ \\
Giardia lamblia & $43(2.3 \%)$ & $139(14.4 \%)$ & $36(11.9 \%)$ & $218(6.9 \%)$ & $<0.003$ \\
Total sample & 1887 & 963 & 302 & 3152 & \\
\hline
\end{tabular}

Prevalence of intestinal helminth infections was higher in females, 55.7\% (95CI\%, 51.7$59.7, \mathrm{n}=642)$ than males $(\mathrm{n}=511,44.32 \%, 95 \% \mathrm{CI}, 40.3-48.6)(P<0.002)$ (Table 2). The prevalence of protozoan infections was 20.5\% (95\%CI, 17.5-23.6, n=646) and it was significantly higher among females $(58.1 \%, 95 \% \mathrm{CI}, 53.1-63.1)$ than males $(42.3 \%, 95 \% \mathrm{CI}$, 36.5-48.2) (Table 2) $(P<0.005)$.

Table 2: Prevalence (\%) of helminth and protozoan infections stratified by sex

\begin{tabular}{lllll}
\hline Parasite & Males & Females & Overall prevalence & P-value \\
\hline Hookworm & $359(24.9 \%)$ & $434(25.4 \%)$ & $793(25.2 \%)$ & $<0.005$ \\
A. lumbricoides & $27(1.9 \%)$ & $23(1.3 \%)$ & $50(1.6 \%)$ & $<0.007$ \\
E. vermicularis & $12(0.8 \%)$ & $9(0.5 \%)$ & $21(0.7 \%)$ & $<0.06$ \\
T. trichiura & $12(0.8 \%)$ & $13(0.8 \%)$ & $25(0.8 \%)$ & $<0.06$ \\
S. mansoni & $93(6.5 \%)$ & $84(4.9 \%)$ & $177(5.6 \%)$ & $<0.05$ \\
Taenia species & $8(0.6 \%)$ & $10(0.6 \%)$ & $18(0.6 \%)$ & $<0.06$ \\
E. histolytica/E. dispar & $174(12 \%)$ & $254(14.9 \%)$ & $428(13.5 \%)$ & $<0.002$ \\
G. lamblia/G. intestinalis & $99(6.9 \%)$ & $110(6.4 \%)$ & $209(6.6 \%)$ & $<0.003$
\end{tabular}


Age group specific prevalence of helminthes was highest among 45+ years olds and lowest in the 0-4 years olds (Table 3). Age-specific prevalence for protozoan infections were highest in the 5-14 years age group $(30.9 \%, 95 \% \mathrm{CI}, 27.9-33.8)$ and lowest in the $0-4$ years age group (Table 3 ).

Table 3: Prevalence (in \%) of helminth and protozoan infections stratified by age

\begin{tabular}{lllll}
\hline Type infection & \multicolumn{4}{l}{ Age groups (Years) } \\
& $0-4$ & $5-14$ & $15-44$ & $45+$ \\
\hline Helminth & & & & \\
Hookworm & $10.4(36)$ & $21.1(71)$ & $26.6(509)$ & $32.0(177)$ \\
A. lumbricoides & $1.5(5)$ & $2.1(7)$ & $1.7(32)$ & $1.1(6)$ \\
E. vermicularis & $0(0)$ & $0.6(2)$ & $0.7(13)$ & $1.1(6)$ \\
T. trichiura & $0.6(2)$ & $1.8(6)$ & $0.7(13)$ & $0.5(3)$ \\
S. mansoni & $2.0(7)$ & $5.0(17)$ & $6.5(125)$ & $5.1(28)$ \\
Taenia species & $0.3(1)$ & $0.6(2)$ & $0.5(10)$ & $0.4(2)$ \\
Age group prevalence & $14.8(51)$ & $31.2(105)$ & $36.7(702)$ & $40.1(222)$ \\
Protozoa & & & & \\
E. histolytical E. dispar & $7.5(26)$ & $16.3(55)$ & $14.2(272)$ & $15.4(85)$ \\
Giardia lamblia & $8.1(28)$ & $14.5(49)$ & $4.7(90)$ & $6.2(34)$ \\
Age group prevalence & $15.7(54)$ & $30.9(104)$ & $18.9(362)$ & $21.5(119)$ \\
Total samples & 345 & 337 & 1,914 & 553 \\
\hline
\end{tabular}

\section{Discussion}

In general, the prevalence of intestinal helminthes and protozoan parasites detected in the present study was similar to a previous study in Nigeria (Ozumba \& Ozumba, 2002). The prevalence of intestinal helminth observed in this study, confirm that intestinal helminthiases, mainly hookworm infection, are prevalent among patients attending Bugando Medical Centre, and mostly likely in its catchment area. Previous epidemiological surveys within the Lake Victoria basin have also reported a high prevalence of hookworm infections than other geohelminths (Lwambo et al., 1999; Mazigo et al., 2010b). The higher prevalence of hookworm infections has previously been reported in other rural tropical areas of low socio-economic status (Hotez, 2003; Standley et al., 2010). Similarly, the high prevalence observed in our study likely to be associated with poor hygienic conditions and low socio-economic status of the population in the Lake Victoria basin.

On the other hand, the prevalence of other helminths, A. lumbricoides, E. vermicularis and Trichuris trichiura were very low. Indeed, earlier studies in the nearby Magu district, reported a prevalence of $<1 \%$ of $A$. lumbricoides, Trichuris trichiura and E. vermicularis (Lwambo et al., 1999) while one of our previous studies in the nearby Sengerema district did not detect any A. lumbricoides, T. trichiura or E. vermicularis (Mazigo et al., 2010b). The low prevalence of these parasites is likely to be influenced by the ecological factors within the Lake Victoria basin. Contrary to findings from field 
epidemiological studies, this study observed a low prevalence of S. mansoni (5.6\%). Prevalence between $64 \%$ and $68 \%$ have recently been reported (Standley et al., 2010; Mazigo et al. 2010b). The prevalence of E. histolytical E. dispar and G. lamblia reported in the present study was similar to that reported from Cameroon (Mbuh et al., 2010). The high prevalence of E. histolytica/ E. dispar could be due to the existence of resistant cysts of the parasite in the study area as reported by Mbuh et al. (2010) in a recent study Cameroon.

When the prevalence of intestinal helminthiasis was analysed by sex, the females had a higher frequency of hookworm infections whereas the males had higher frequency of infections by S. mansoni, E. vermicularis and T. trichiura. Such sex predominance in infections rates is likely to be a reflection of different behaviour between the two groups (Albanico et al., 1997; Hotez et al., 2006). In a recent study in Cameroon, it was found that the higher prevalence of human intestinal protozoans in females was attributed to the fact that women usually eat unwashed fruits and vegetables or unboiled salads which may be contaminated with protozoan cysts (Mbuh et al., 2010).

The trend of increasing prevalence of helminths with age observed in this study has been previously reported by other workers in the region (Lwambo et al., 1999; Mazigo et al., 2010b). However, it was different from the normal U-shaped curve reported from a study by Kurup et al. (2010). Our findings suggest that every individual in the region is at high risk of helminth infection. The age relationship in the prevalence of E. histolytical E. dispar and G. lamblia has been reported in various regions of Cameroon (Rossignol et al., 2001; Mbuh et al., 2010).

In conclusion, this study shows that intestinal helminthiases and protozoan infections are among the common parasitic infections observed among patients presenting at BMC. This, and similar information, generated from hospitals may provide invaluable statistics needed for planning meaningful public control programmes that aim at reducing the prevalence and morbidity of parasitic infections.

\section{Acknowledgements}

The authors acknowledge the support of laboratory technicians at the Department of Medical Parasitology, BMC during data collections and cleaning.

\section{References}

Albanico, M., Chwaya, H.M., Montresor, A., Stolfzfus, R.J., Tielsch, J.M., Alawi, K.S. \& Savioli, L. (1997) Parasitic infections in Pemba island schoolchildren. East African Medical Journal 74, 294-298.

Albanico, M., Stoltzfus, R.J. \& Savioli L. (1998) Epidemiological evidence for a differential effect of hookworm species, Ancylostoma duodenale or Necator americanus, on iron status of children. International Journal of Epidemiology 27, 530537. 
Awasti, S., Bundy, D.A.P. \& Savioli L. (2003) Helminthic infections. Brazil Medical Journal 327, 431-433.

Bethony, J., Chen, J. \& Lin, S. (2002) Emerging patterns of hookworm infection: Influence of aging on the intensity of Necator infection in Hainan Province, People's Republic of China. Clinical Infectious Disease 35, 1336-1344.

Bethony, J., Brooker, S., Albanico, M., Geiger, S.M., Loukas, A., Diemert, D. \& Hotez, P.J. (2006) Soil-transmitted helminth infections; Ascaris, Trichuriasis and hookworm. Lancet 367, 1521.

Boros, D.L. (1989) Immunopathology of Schistosoma mansoni infection. Clinical Microbiology Review 2, 250-269.

Garcia, L.S.H. (2004) Diagnostic Medical Parasitology, 4th edition, ASM Press 2004.

Guyatt, H.H., Brooker, S., Lwambo, N.J.S., Siza, J. E., \& Bundy, A. P. (1999) The performance of school-based questionnaires of reported blood in urine in diagnosing S. haematobium infection: Patterns by age and sex. Tropical Medicine and International Health 4, 751-757.

Haidar, N.A. (2001) Schistosoma mansoni as a cause of bloody stool in children. Saudia Medical Journal 22, 856-859.

Handzel, T., Karanja, D.M., Addiss, D.G., Hightower, A.W., Rosen, D.H., Colley, D.G., Andove, J., Slutsker, L. \& Evansecor, W. (2003) Geographic distribution of schistosomiasis and soil-transmitted helminths in Western Kenya: Implication for anthelminthic mass treatment. American Journal of Tropical Medicine and Hygiene 69, 318-323

Hotez, P.J., Brooker, S., Bethony, J.M., Bottazzi, M.E., Loukas, A. \& Xiao S. (2004) Hookworm infection. New England Journal of Medicine, 351, 799-807.

Hotez, P.J. (2003) Hookworm in the Americans. Progress in the development of an antihookworm vaccine. In de Quadros CA. editor. Vaccines: Preventing Disease and Protecting Health. Washington (D.C.). Pan American Health Organization 213-223.

Hotez, P.J., Bundy, D.A.P. \& Beegle K. (2006) Helminth infections. Soil-Transmitted Infections and Schistosomiasis. The International Bank for Reconstruction and Development/The World Bank, 2006.

Kurup, R. \& Hunjan, G.S. (2010) Intestinal parasites in St Lucia: A retrospective, laboratory-based study. Journal of Rural and Tropical Public Health 9, 24-30.

Lwambo, N. J. S., Siza, J. E., Brooker, S., Bundy, D. A. P. \& Guyatt, H. (1999) Patterns of concurrent hookworm infection and schistosomiasis in schoolchildren in Tanzania. Transactions of the Royal Society of Tropical Medicine and Hygiene 93, 497502.

Malenganisho, W.L.M., Magnussen, P., Friis, H., Siza, J., Kaatano, G., Temu, M. \& Vennervald, B.J. (2008) Schistosoma mansoni morbidity among adults in two villages along Lake Victoria shores in Mwanza District, Tanzania. Transactions of the Royal Society of Tropical Medicine and Hygiene 102, 532-541.

Mazigo, H.D., Lwambo, N.J.S., Mkoji, G.M., Laurent, L.M., Kweka, E.J. \& R Waihenya, R. (2010a) Anaemia and organomegaly associated with parasitic infections 
among schoolchildren in Sengerema District, north--western Tanzania. Tanzania Journal of Health Research 12 (in press).

Mazigo, H.D.,Waihenya, R., Lwambo,N.J.S., Mnyone, L.L., Mahande, A.M., Seni, J., Zinga, M., Kapesa, A., Kweka, E.J., Mshana, S.E., Heukelbach, J. \& Mkoji, G.M. (2010b) Co-infections with Plasmodium falciparum, Schistosoma mansoni and intestinal helminth among schoolchildren in endemic areas of northwestern Tanzania. BMC Parasites and Vectors 3:44

Mbuh, J.V., Ntonifor, H.N. \& Ojong, J.T. (2010) The incidence, intensity and host morbidity of human parasitic protozoan infections in gastrointestinal disorder outpatients in Buea Sub Division, Cameroon. Journal of Infection in Developing Countries 4, 38-43.

Obiamiwe, B.A. (1972) The pattern of parasitic infection in human gut at the specialist hospital Benin City, Nigeria. Annals of Tropical Medicine and Parasitology 71, 35-43.

Ozumba, U.C. \& Ozumba, N. (2002) Patterns of helminth infection in the human gut at the University of Nigeria Teaching Hospital, Enugu, Nigeria. Journal of Health Science, 48, 263-268.

Ravdin, J.I. (1995) Amoebiasis. Clinical Infectious Disease 20, 1453-1466.

Rossignol, J.F., Ayoub, A. \& Ayers, M.S. (2001) Treatment of diarrhoea caused by Giardia intestinalis and Entamoeba histolytical E. dispar: A randomized, double blind, Placebo-controlled study of Nitaxoxanide. Journal of Infectious Diseases 184, 381384.

Sayyari, A.A., Imanzadeh, F., Bagheri, Y.S.A., Karami, H. \& Yaghoobi, M. (2005) Prevalence of intestinal parasitic infections in the Islamic Republic of Iran. East Mediterranean Health Journal 11, 377-383.

Standley, C.J., Lwambo, N.J.S., Lange, C.N., Kariuki, H.C., Adriko M. \& Stothard, J.R. (2010) Performance of circulating cathodic antigen (CCA) urine-dipsticks for rapid detection of intestinal schistosomiasis in schoolchildren from shoreline communities of Lake Victoria. BMC Parasite and Vector 3:7.

WHO (1991) Basic Laboratory Methods in Medical Parasitology. World Health Organization, Geneva. 